\title{
508.
}

\section{ON GEODESIC LINES, IN PARTICULAR THOSE OF A QUADRIC SURFACE.}

[From the Proceedings of the London Mathematical Society, vol. IV. (1871-1873), pp. 191-211. Read December 12, 1872.]

The present Memoir contains an investigation of the differential equation (of the second order) of the geodesic lines on a surface, the coordinates of a point on the surface being regarded as given functions of two parameters $p$, $q$, and researches in connection therewith; a deduction of Jacobi's differential equation of the first order in the case of a quadric surface, the parameters $p, q$ being those which determine the two sets of curves of curvature; formulæ where the parameters are those which determine the two right lines through the surface; and a discussion of the forms of the geodesic lines in the two cases of an ellipsoid and a skew hyperboloid respectively.

\section{Preliminary Formulce.}

1. I call to mind the fundamental formulæ in the Memoir by Gauss, "Disquisitiones generales circa superficies curvas," Comm. Gott. recent. t. VI., 1827, (reprinted as an Appendix in Liouville's edition of Monge,) together with some that I have added to them. The coordinates $x, y, z$ of a point on a surface are regarded as given functions of two parameters $p, q$, these expressions of $x, y, z$ in effect determining the equation of the surface, and we have

$$
\begin{gathered}
d x+\frac{1}{2} d^{2} x=a d p+a^{\prime} d q+\frac{1}{2}\left(\alpha d p^{2}+2 \alpha^{\prime} d p d q+\alpha^{\prime \prime} d q^{2}\right), \\
d y+\frac{1}{2} d^{2} y=b d p+b^{\prime} d q+\frac{1}{2}\left(\beta d p^{2}+2 \beta^{\prime} d p d q+\beta^{\prime \prime} d q^{2}\right), \\
d z+\frac{1}{2} d^{2} z=c d p+c^{\prime} d q+\frac{1}{2}\left(\gamma d p^{2}+2 \gamma^{\prime} d p d q+\gamma^{\prime \prime} d q^{2}\right), \\
A, B, C=b c^{\prime}-b^{\prime} c, c a^{\prime}-c^{\prime} a, a b^{\prime}-a^{\prime} b
\end{gathered}
$$


whence differential equation of surface is

$$
A d x+B d y+C d z=0 .
$$

Also

$$
E, F, G=a^{2}+b^{2}+c^{2}, \quad a a^{\prime}+b b^{\prime}+c c^{\prime}, \quad a^{\prime 2}+b^{\prime 2}+c^{\prime 2} ;
$$

so that element of length on the surface is given by

or, as I write it,

$$
d x^{2}+d y^{2}+d z^{2}=E d p^{2}+2 F d p d q+G d q^{2} ;
$$

and moreover

$$
=(E, F, G \gamma d p, d q)^{2} ;
$$

$$
V^{2}=A^{2}+B^{2}+C^{2}=E G-F^{2} .
$$

The equation $(E, F, G \gamma d p, d q)^{2}=0$ determines at each point on the surface two directions (necessarily imaginary) which are called the "circular" directions. Passing on the surface from point to point along the circular directions, we obtain two series of curves (always imaginary) which are the "circular" curves; the equation $(E, F, G \gamma d p, d q)^{2}=0$ is the differential equation of these curves; and if we have $E=0, G=0$, then this becomes $d p d q=0$; viz. we have in this case $p=$ const. and $q=$ const. as the equations of the two sets of circular curves respectively. It is clear $\grave{a}$ priori, and will be shown analytically in the sequel, that the circular curves are geodesic lines.

I write also

$$
E^{\prime}, F^{\prime \prime}, G^{\prime}=A \alpha+B \beta+C \gamma, \quad A \alpha^{\prime}+B \beta^{\prime}+C \gamma^{\prime}, \quad A \alpha^{\prime \prime}+B \beta^{\prime \prime}+C \gamma^{\prime \prime},
$$

or, what is the same thing, $E^{\prime}, F^{\prime}, G^{\prime}$ represent the determinants

$$
\left|\begin{array}{lll}
a, & b, & c \\
a^{\prime}, & b^{\prime}, & c^{\prime} \\
\alpha, & \beta, & \gamma
\end{array}\right|,\left|\begin{array}{lll}
a, & b, & c \\
a^{\prime}, & b^{\prime}, & c^{\prime} \\
\alpha^{\prime}, & \beta^{\prime}, & \gamma^{\prime}
\end{array}\right|,\left|\begin{array}{lll}
a, & b, & c \\
a^{\prime}, & b^{\prime}, & c^{\prime} \\
\alpha^{\prime \prime}, & \beta^{\prime \prime}, & \gamma^{\prime \prime}
\end{array}\right| \text {, respectively, }
$$

(these last symbols do not occur in Gauss). [They are the $D, D^{\prime}, D^{\prime \prime}$ of Gauss.]

2. The radius of curvature of normal section corresponding to direction $d p: d q$ is given by

$$
\frac{\rho}{V}=\frac{(E, F, G \gamma d p, d q)^{2}}{\left(E^{\prime}, F^{\prime}, G^{\prime} \chi d p, d q\right)^{2}}
$$

whence it appears that the directions of the inflexional or chief tangents (the Haupttangenten) are determined by the equation

$$
\left(E^{\prime}, F^{\prime}, G^{\prime} \chi d p, d q\right)^{2}=0 .
$$

The directions in question are imaginary on a surface such as the ellipsoid where the curvatures are in the same direction, but on a concavo-convex surface they are real; and in particular on the hyperboloid they coincide with the directions of the generating lines. We may on any surface pass from point to point along the chief directions; we have thus on the surface two sets of curves which are the chief curves; 
the differential equation of these is $\left(E^{\prime}, F^{\prime}, G^{\prime} \gamma d p, d q\right)^{2}=0$; and in particular if $E^{\prime}=0, G^{\prime}=0$, then this becomes $d p d q=0$, or we have $p=$ const., $q=$ const. for the equations of the two sets of chief curves respectively. On the hyperboloid the chief curves are the two sets of generating lines. The chief curves are not in general geodesic lines, but on the hyperboloid, quà straight lines, they are, it is clear, geodesic lines.

3. The directions of the curves of curvature, or the principal tangents, and the corresponding values of the radius of curvature are determined by

$$
\frac{\rho}{V}=\frac{E d p+F d q}{E^{\prime} d p+F^{\prime} d q}=\frac{F d p+G d q}{F^{\prime} d p+G^{\prime} d q}
$$

or, what is the same thing, these directions are determined by the equation

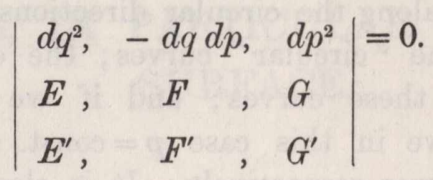

The same equations may be written

$$
-d q: d p=\frac{\rho E^{\prime}-V E}{\rho F^{\prime}-V F}=\frac{\rho F^{\prime}-V F}{\rho G^{\prime}-V G}
$$

that is the principal radii of curvature are determined by the equation

$$
\rho^{2}\left(E^{\prime} G^{\prime}-F^{\prime 2}\right)-\rho V\left(E G^{\prime}+E^{\prime} G-2 F F^{\prime}\right)+V^{2}\left(E G-F^{2}\right)=0,
$$

(last term is $=V^{4}$, but it is better to retain the original form): and then, $\rho$ being either root, the last preceding equations give the direction of the curve of curvature corresponding to the given value of the radius of curvature.

If $p=$ const., $q=$ const. are the equations of the two systems of curves of curvature respectively, then the quadric equation in $(d p, d q)$ must become $d p d q=0$; this will be so if $F=0, F^{\prime}=0$; and we thus have these equations, viz. written at full length they are

$$
\begin{aligned}
& d_{p} x d_{q} x+d_{p} y d_{q} y+d_{p} z d_{q} z=0,
\end{aligned}
$$

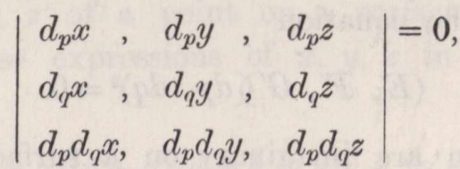

as the conditions in order that $p=$ const., $q=$ const. may be the two systems of curves of curvature. The former of these equations merely expresses that the two sets of curves always intersect at right angles. 


\section{General Theory of the Geodesic Lines on a Surface.}

4. I now proceed to investigate the theory of geodesic lines on a surface, the surface being determined as above by means of given expressions of the coordinates $x, y, z$ in terms of the parameters $p, q$.

The differential equation obtained by Gauss for the geodesic lines is in a form not symmetrical in regard to the two variables; viz. his equation is

where, as above,

$$
\frac{d E}{d q} d p^{2}+2 \frac{d F}{d p} d p d q+\frac{d G}{d p} d p^{2}=2 d s d \frac{E d p+F d q}{d s},
$$

$$
d s^{2}=(E, F, G \gamma d p, d q)^{2} .
$$

If we herein consider $p, q$ as functions of a parameter $\theta$, and write for shortness

also

$$
d_{\theta} p, d_{\theta} q, d_{\theta}^{2} p, \& c .=p^{\prime}, q^{\prime}, p^{\prime \prime}, \& c .
$$

$$
\Omega=\left(E, F, G \gamma\left(p^{\prime}, q^{\prime}\right)^{2},\right.
$$

and

then the equation is

$$
d_{p} E=E_{1}, \quad d_{q} E=E_{2}, \& c .
$$

$$
\left(E_{1}, F_{1}, G_{1} \gamma p^{\prime}, q^{\prime}\right)^{2}-2 \sqrt{ } \Omega\left(\frac{E p^{\prime}+F q^{\prime}}{\sqrt{ } \Omega}\right)^{\prime}=0 .
$$

We have

$$
\left(\frac{E p^{\prime}+F q^{\prime}}{\sqrt{ } \Omega}\right)^{\prime}=\frac{1}{\Omega \sqrt{ } \Omega}(M+N)
$$

where $N$ is the part containing $p^{\prime \prime}, q^{\prime \prime}$, which I will first calculate; viz. we have

$$
\begin{aligned}
N & =\Omega\left(E p^{\prime \prime}+F q^{\prime \prime}\right)-\left(E p^{\prime}+F q^{\prime}\right) \frac{1}{2} \Omega^{\prime}, \\
& =\Omega\left(E p^{\prime \prime}+F q^{\prime \prime}\right)-\left(E p^{\prime}+F q^{\prime}\right)\left\{\left(E p^{\prime}+F q^{\prime}\right) p^{\prime \prime}+\left(F p^{\prime}+G q^{\prime}\right) q^{\prime \prime}\right\}, \\
& =p^{\prime \prime}\left\{E \Omega-\left(E p^{\prime}+F q^{\prime}\right)^{2}\right\}+q^{\prime \prime}\left\{F \Omega-\left(E p^{\prime}+F q^{\prime}\right)\left(F p^{\prime}+G q^{\prime}\right)\right\} ;
\end{aligned}
$$

or substituting for $\Omega$ its value, this is

$$
=p^{\prime \prime}\left(E G-F^{2}\right) q^{\prime 2}-q^{\prime \prime}\left(E G-F^{2}\right) p^{\prime} q^{\prime}, \quad=-q^{\prime}\left(E G-F^{2}\right)\left(p^{\prime} q^{\prime \prime}-p^{\prime \prime} q^{\prime}\right) ;
$$

wherefore

$$
\left(\frac{E p^{\prime}+F q^{\prime}}{\sqrt{ } \Omega}\right)^{\prime}=\frac{1}{\Omega \sqrt{ } \Omega}\left\{M-q^{\prime}\left(E G-F^{2}\right)\left(p^{\prime} q^{\prime \prime}-p^{\prime \prime} q^{\prime}\right)\right\},
$$

and the equation becomes

$$
\Omega\left(E_{1} p^{\prime 2}+2 F_{1} p^{\prime} q^{\prime}+G_{1} q^{\prime 2}\right)-2 M+2 q^{\prime}\left(E G-F^{2}\right)\left(p^{\prime} q^{\prime \prime}-p^{\prime \prime} q^{\prime}\right)=0 ;
$$

whence we foresee that the whole equation must divide by $q^{\prime}$. 
5. We have

$$
\begin{aligned}
M= & \left(p^{\prime} d E+q^{\prime} d F\right) \Omega-\left(E p^{\prime}+F q^{\prime}\right)\left(\frac{1}{2} p^{\prime 2} d E+p^{\prime} q^{\prime} d F+\frac{1}{2} q^{\prime 2} d G\right) \\
= & d E\left\{p^{\prime} \Omega-\frac{1}{2} p^{\prime 2}\left(E p^{\prime}+F q^{\prime}\right)\right\} \\
& +d F\left\{q^{\prime} \Omega-p^{\prime} q^{\prime}\left(E p^{\prime}+F q^{\prime}\right)\right\} \\
& +d G\left\{-\frac{1}{2} q^{\prime 2}\left(E p^{\prime}+F q^{\prime}\right)\right\}
\end{aligned}
$$

or say

$$
\begin{aligned}
2 M= & d E\left[p^{\prime 2}\left(p^{\prime} E+q^{\prime} F^{\prime}\right)+2 p^{\prime} q^{\prime}\left(p^{\prime} F+q^{\prime} G\right)\right] \\
& +d F^{\prime}\left[\quad 2 q^{\prime 2}\left(p^{\prime} F+q^{\prime} G\right)\right] \\
& +d G\left[-q^{\prime 2}\left(p^{\prime} E+q^{\prime} F^{\prime}\right)\right. \\
= & \left(E_{1} p^{\prime}+E_{2} q^{\prime}\right)\left[p^{\prime 2}\left(E p^{\prime}+F q^{\prime}\right)+2 p^{\prime} q^{\prime}\left(F p^{\prime}+G q^{\prime}\right)\right] \\
& +\left(F_{1}^{\prime} p^{\prime}+F_{2} q^{\prime}\right)[ \\
& +\left(G_{1} p^{\prime}+G_{2} q^{\prime}\right)\left[-q^{\prime 2}\left(E p^{\prime}+G q^{\prime}\right)\right] \\
&
\end{aligned}
$$

The term in $p^{\prime 4}$ is $E E_{1} p^{\prime 4}$, which is also the term in $p^{\prime 4}$ of $\Omega\left(E_{1} p^{\prime 2}+2 F_{1} p^{\prime} q^{\prime}+G_{1} q^{\prime 2}\right)$; whence $\Omega\left(E_{1} p^{\prime 2}+2 F_{1} p^{\prime} q^{\prime}+G_{1} q^{\prime 2}\right)-2 M$ divides by $q^{\prime}$.

Proceeding to the reduction:

Term in $E_{1}$ is

$$
E_{1} \cdot p^{\prime 2} \Omega-p^{\prime 3}\left(E p^{\prime}+F q^{\prime}\right)-2 p^{\prime 2} q^{\prime}\left(F p^{\prime}+G q^{\prime}\right),=E_{1} \cdot-p^{\prime 2} q^{\prime}\left(F p^{\prime}+G q^{\prime}\right)
$$

term in $F_{1}$ is

$$
F_{1} \cdot 2 p^{\prime} q^{\prime} \Omega-2 p^{\prime} q^{\prime 2}\left(F p^{\prime}+G q^{\prime}\right),=F_{1} \cdot 2 p^{\prime 2} q^{\prime}\left(E p^{\prime}+F q^{\prime}\right)
$$

term in $G_{1}$ is

$$
G_{1} \cdot q^{\prime 2} \Omega+p^{\prime} q^{\prime 2}\left(E p^{\prime}+F q^{\prime}\right),=G_{1}\left\{2 p^{\prime} q^{\prime 2}\left(E p^{\prime}+F q^{\prime}\right)+q^{\prime 3}\left(F p^{\prime}+G q^{\prime}\right)\right\} .
$$

6. The remaining terms in $E_{2}, F_{2}, G_{2}$ require no reduction, and the result is

$$
\begin{aligned}
& E_{1}\left\{-p^{\prime 2}\left(F p^{\prime}+G q^{\prime}\right)\right\}-E_{2}\left\{p^{2}\left(E p^{\prime}+F q^{\prime}\right)+2 p^{\prime} q^{\prime}\left(F p^{\prime}+G q^{\prime}\right)\right\} \\
+ & F_{1}\left\{2 p^{\prime 2}\left(E p^{\prime}+F q^{\prime}\right)\right\}-F_{2}\left\{2 q^{\prime 2}\left(F p^{\prime}+G q^{\prime}\right)\right\} \\
+ & G_{1}\left\{2 p^{\prime} q^{\prime}\left(E p^{\prime}+F q^{\prime}\right)+q^{\prime 2}\left(F p^{\prime}+G q^{\prime}\right)\right\}-G_{2}\left\{-q^{\prime 2}\left(E p^{\prime}+F q^{\prime}\right)\right\} \\
& +2\left(E G-F^{2}\right)\left(p^{\prime} q^{\prime \prime}-p^{\prime \prime} q^{\prime}\right)=0,
\end{aligned}
$$

or, what is the same thing,

$$
\begin{aligned}
\left(E p^{\prime}+F q^{\prime}\right)\left\{\left(2 F_{1}-E_{2}\right) p^{\prime 2}+2 G_{1} p^{\prime} q^{\prime}+G_{2} q^{\prime 2}\right\} \\
-\left(F p^{\prime}+G q^{\prime}\right)\left\{E_{1} p^{\prime 2}+2 E_{2} p^{\prime} q^{\prime}+\left(2 F_{2}-G_{1}\right) q^{\prime 2}\right\} \\
+2\left(E G-F^{2}\right)\left(p^{\prime} q^{\prime \prime}-p^{\prime \prime} q^{\prime}\right)=0,
\end{aligned}
$$

which is the required differential equation of the second order: the independent variable has been taken to be the arbitrary quantity $\theta$; but taking $\theta=p$, or $q$, say $\theta=p$, we have $p^{\prime}=1, p^{\prime \prime}=0$, and the equation then contains (besides $p$ and $q$ ) only $q^{\prime \prime}$ and $q^{\prime}$, that is, $d_{p}{ }^{2} q$. and $d_{p} q$, and is therefore a differential relation between $p$ and $q$. 
7. Instead of starting, as above, from the equation given by Gauss, we may use the geometrical property that at each point of a geodesic line the osculating plane passes through the normal of the surface.

Considering, as above, $p, q$ as functions of a variable $\theta$, then, $\theta$ becoming $\theta+d \theta$, the new values of $p, q$ are

and that of $x$ is

$$
p+p^{\prime} d \theta+\frac{1}{2} p^{\prime \prime} d \theta^{2}, \quad q+q^{\prime} d \theta+\frac{1}{2} q^{\prime \prime} d \theta^{2} ;
$$

$$
x+a\left(p^{\prime} d \theta+\frac{1}{2} p^{\prime \prime} d \theta^{2}\right)+a^{\prime}\left(q^{\prime} d \theta+\frac{1}{2} q^{\prime \prime} d \theta^{2}\right)+\frac{1}{2}\left(\alpha p^{\prime 2}+2 \alpha^{\prime} p^{\prime} q^{\prime}+\alpha^{\prime \prime} q^{\prime 2}\right) d \theta^{2} ;
$$

or calling this $x+x^{\prime} d \theta+\frac{1}{2} x^{\prime \prime} d \theta^{2}$, we have

and so

$$
x^{\prime}=a p^{\prime}+a^{\prime} q^{\prime}, \quad x^{\prime \prime}=a p^{\prime \prime}+a^{\prime} q^{\prime \prime}+\alpha p^{\prime 2}+2 \alpha^{\prime} p^{\prime} q^{\prime}+\alpha^{\prime \prime} q^{\prime 2},
$$

$$
\begin{array}{ll}
y^{\prime}=b p^{\prime}+b^{\prime} q^{\prime}, & y^{\prime \prime}=b p^{\prime \prime}+b^{\prime} q^{\prime \prime}+\beta p^{\prime 2}+2 \beta^{\prime} p^{\prime} q^{\prime}+\beta^{\prime \prime} q^{\prime 2}, \\
z^{\prime}=c p^{\prime}+c^{\prime} q^{\prime}, & z^{\prime \prime}=c p^{\prime \prime}+c^{\prime} q^{\prime \prime}+\gamma p^{2}+2 \gamma^{\prime} p^{\prime} q^{\prime}+\gamma^{\prime \prime} q^{\prime 2} .
\end{array}
$$

The condition in question is expressed by the equation

$$
\left|\begin{array}{ccc}
A, & B, & C \\
x^{\prime}, & y^{\prime}, & z^{\prime} \\
x^{\prime \prime}, & y^{\prime \prime}, & z^{\prime \prime}
\end{array}\right|=0,
$$

that is

$$
+\left|\begin{array}{ccc}
A, & B, & C \\
A, & \begin{array}{ccc}
a p^{\prime}+a^{\prime} q^{\prime}, & b p^{\prime}+b^{\prime} q^{\prime}, & c p^{\prime}+c^{\prime} q^{\prime} \\
a p^{\prime \prime}+a^{\prime} q^{\prime \prime}, & b p^{\prime \prime}+b^{\prime} q^{\prime \prime}, & c p^{\prime \prime}+c^{\prime} q^{\prime \prime}
\end{array} \mid \\
a p^{\prime}+a^{\prime} q^{\prime}, & B, & C \\
a p^{\prime 2}+2 \alpha^{\prime} p^{\prime} q^{\prime}+\alpha^{\prime \prime} q^{\prime 2}, & \beta p^{\prime 2}+2 \beta^{\prime} p^{\prime} q^{\prime}+\beta^{\prime \prime} q^{\prime 2}, & \gamma p^{\prime 2}+\gamma^{\prime} p^{\prime} q^{\prime}+\gamma^{\prime \prime} q^{\prime 2}
\end{array}\right|=0 .
$$

8. The first determinant is the sum of three terms such as $A\left(b c^{\prime}-b^{\prime} c\right)\left(p^{\prime} q^{\prime \prime}-p^{\prime \prime} q^{\prime}\right)$; viz. this is $A^{2}\left(p^{\prime} q^{\prime \prime}-p^{\prime \prime} q^{\prime}\right)$, or the determinant is

$$
\left(A^{2}+B^{2}+C^{2}\right)\left(p^{\prime} q^{\prime \prime}-p^{\prime \prime} q^{\prime}\right),=\left(E G-F^{2}\right)\left(p^{\prime} q^{\prime \prime}-p^{\prime \prime} q^{\prime}\right) .
$$

The second determinant is the sum of three terms such as

$$
\left(\alpha p^{\prime 2}+2 \alpha^{\prime} p^{\prime} q^{\prime}+\alpha^{\prime \prime} q^{\prime 2}\right)\left[B\left(c p^{\prime}+c^{\prime} q^{\prime}\right)-C\left(b p^{\prime}+b^{\prime} q^{\prime}\right)\right],
$$

where the factor in [ ] is

$$
p^{\prime}\left[c\left(c a^{\prime}-c^{\prime} a\right)-b\left(a b^{\prime}-a^{\prime} b\right)\right]+q^{\prime}\left[c^{\prime}\left(c a^{\prime}-c^{\prime} a\right)-b^{\prime}\left(a b^{\prime}-a^{\prime} b\right)\right],
$$

C. VIII. 
which is $=p^{\prime}\left(a^{\prime} E-a F^{\prime}\right)+q^{\prime}\left(a^{\prime} F-a G\right)$. We have thus the second determinant, and the equation becomes

$$
\begin{aligned}
(E G- & \left.F^{2}\right)\left(p^{\prime} q^{\prime \prime}-p^{\prime \prime} q^{\prime}\right) \\
& +\left(\alpha p^{\prime 2}+2 \alpha^{\prime} p^{\prime} q^{\prime}+\alpha^{\prime \prime} q^{\prime 2}\right)\left\{p^{\prime}\left(a^{\prime} E-a F\right)+q^{\prime}\left(a^{\prime} F-a G\right)\right\} \\
& +\left(\beta p^{\prime 2}+2 \beta^{\prime} p^{\prime} q^{\prime}+\beta^{\prime \prime} q^{\prime 2}\right)\left\{p^{\prime}\left(b^{\prime} E-b F\right)+q^{\prime}\left(b^{\prime} F-b G\right)\right\} \\
& +\left(\gamma p^{\prime 2}+2 \gamma^{\prime} p^{\prime} q^{\prime}+\gamma^{\prime \prime} q^{\prime 2}\right)\left\{p^{\prime}\left(c^{\prime} E-c F\right)+q^{\prime}\left(c^{\prime} F-c G\right)\right\}=0,
\end{aligned}
$$

an equation of the same form as that previously obtained, and which can be without difficulty identified therewith.

\section{The Circular Curves are Geodesics.}

9. I proceed to show that the circular curves are geodesics; viz. that an integral of the geodesic equation is

$$
\left(E, F, G \gamma p^{\prime}, q^{\prime}\right)^{2}=0 .
$$

Starting from this equation, we have

$2\left\{\left(E p^{\prime}+F q^{\prime}\right) p^{\prime \prime}+\left(F p^{\prime}+G q^{\prime}\right) q^{\prime \prime}\right\}+\left(E_{1} p^{\prime}+E_{2} q^{\prime}\right) p^{\prime 2}+2\left(F_{1} p^{\prime}+F_{2} q^{\prime}\right) p^{\prime} q^{\prime}+\left(G_{1} p^{\prime}+G_{2} q^{\prime}\right) q^{\prime 2}=0$.

Now the equation, writing therein $E p^{\prime}+F q^{\prime}=\lambda q^{\prime}$, gives $F p^{\prime}+G q^{\prime}=-\lambda p^{\prime}$ : these equations may be written

$$
\begin{aligned}
E p^{\prime}+(F-\lambda) q^{\prime} & =0 \\
(F+\lambda) p^{\prime}+G \quad q^{\prime} & =0
\end{aligned}
$$

the value of $\lambda$ being therefore $-\lambda^{2}=E G-F^{2}$. The result just obtained thus becomes

$$
\begin{aligned}
2 \lambda\left(p^{\prime \prime} q^{\prime}\right. & \left.-p^{\prime} q^{\prime \prime}\right) \\
& +\left[\left(E_{1} p^{\prime}+E_{2} q^{\prime}\right) p^{\prime}+\left(F_{1} p^{\prime}+F_{2} q^{\prime}\right) q^{\prime}\right] \cdot-\frac{1}{\lambda}\left(F p^{\prime}+G q^{\prime}\right) \\
& +\left[\left(F_{1} p^{\prime}+F_{2} q^{\prime}\right) p^{\prime}+\left(G_{1} p^{\prime}+G_{2} q^{\prime}\right) q^{\prime}\right] \cdot \frac{1}{\lambda}\left(E p^{\prime}+F q^{\prime}\right)
\end{aligned}
$$

that is

$$
\begin{aligned}
2(E G- & \left.F^{2}\right)\left(p^{\prime} q^{\prime \prime}-p^{\prime \prime} q^{\prime}\right) \\
& -\left(F p^{\prime}+G q^{\prime}\right)\left[E_{1} p^{\prime 2}+\left(E_{2}+F_{1}\right) p^{\prime} q^{\prime}+F_{2} q^{\prime 2}\right] \\
& +\left(E p^{\prime}+F q^{\prime}\right)\left[F_{1} p^{\prime 2}+\left(F_{2}+G_{1}\right) p^{\prime} q^{\prime}+G_{2} q^{\prime 2}\right]=0
\end{aligned}
$$

or what is the same thing, adding hereto the zero value

$$
\Delta\left(E, F, G \gamma p^{\prime}, q^{\prime}\right)^{2},=\left(F p^{\prime}+G q^{\prime}\right) \Delta q^{\prime}+\left(E p^{\prime}+F q^{\prime}\right) \Delta p^{\prime},
$$

where $\Delta$ is arbitrary, the equation is

$$
\begin{aligned}
2(E G- & \left.F^{2}\right)\left(p^{\prime} q^{\prime \prime}-p^{\prime \prime} q^{\prime}\right) \\
& +\left(E p^{\prime}+F^{\prime} q^{\prime}\right)\left[F_{1}^{\prime} p^{\prime 2}+\left(F_{2}+G_{1}\right) p^{\prime} q^{\prime}+G_{2} q^{\prime 2}+\Delta p^{\prime}\right] \\
& -\left(F p^{\prime}+G q^{\prime}\right)\left[E_{1} p^{\prime 2}+\left(E_{2}+F_{1}\right) p^{\prime} q^{\prime}+F_{2}^{\prime} q^{\prime 2}-\Delta q^{\prime}\right]=0
\end{aligned}
$$

viz. taking $\Delta=\left(F_{1}-E_{2}\right) p^{\prime}+\left(G_{1}-F_{2}\right) q^{\prime}$, this agrees with the geodesic equation.

The foregoing integral, $\left(E, F, G \gamma\left(p^{\prime}, q^{\prime}\right)^{2}=0\right.$, is, I believe, a particular, not a singular, solution of the geodesic equation. 
The Chief Lines are not in general Geodesics.

10. That the chief lines are not in general geodesics appears most readily as follows :

To find the condition in order that $p=$ const. may be a geodesic, we write in the geodesic equation $p^{\prime}=0, p^{\prime \prime}=0$ : the equation thus becomes

that is we have

$$
F q^{\prime} \cdot G_{2} q^{\prime 2}-G q^{\prime}\left(2 F_{2}-G_{1}\right) q^{\prime 2}=0 ;
$$

$$
F G_{2}-2 F_{2} G+G G_{1}=0
$$

as the condition in order that $p=$ const. may be a geodesic: the condition that it may be a chief curve is $G^{\prime}=0$, which is a different condition.

We have of course, in like manner,

$$
2 E F_{1}-E_{1} F-E E_{2}=0
$$

as the condition in order that $q=$ const. may be a geodesic; and $E^{\prime}=0$ as the condition that this may be a chief curve. If $p=$ const., $q=$ const. are each of them at once a geodesic and a chief curve, then the four equations must all be satisfied, viz. we must have

$$
\begin{aligned}
F G_{2}-2 F_{2} G+G G_{1} & =0, & 2 E F_{1}-E_{1} F-E E_{2} & =0 \\
G^{\prime} & =0, & E^{\prime} & =0 .
\end{aligned}
$$

\section{Special Form of the Geodesic Equation.}

11. In the case where the curves $p=$ const., $q=$ const. intersect at right angles (and in particular when these are the curves of curvature), we have $F^{\prime}=0$; whence also $F_{1}=0, F_{2}=0$; and the geodesic equation assumes the more simple form,

$$
\begin{aligned}
& E p^{\prime}\left(-E_{2} p^{\prime 2}+2 G_{1} p^{\prime} q^{\prime}+G_{2} q^{\prime 2}\right) \\
&-G q^{\prime}\left(E_{1} p^{\prime 2}+2 E_{2} p^{\prime} q^{\prime}-G_{1} q^{\prime 2}\right) \\
&+2 E G\left(p^{\prime} q^{\prime \prime}-p^{\prime \prime} q^{\prime}\right)=0 .
\end{aligned}
$$

$\left[11^{a}\right.$. In the case of a surface of revolution we have

$$
x=p \cos q, \quad y=p \sin q, \quad z=p ;
$$

$E$ is of the form $1+P^{\prime 2}, P^{\prime}=d_{p} P$, where $P$ is a function of $p$ only, and we have

that is

$$
d s^{2}=\left(1+P^{\prime 2}\right) d p^{2}+p^{2} d q^{2}
$$

$$
\begin{aligned}
& E=1+P^{2}, \quad E_{1}=2 P^{\prime} P^{\prime \prime}, \quad E_{2}=0, \\
& F=0, \\
& G=p^{2} \quad, G_{1}=2 p \quad, \quad G_{2}=0 ;
\end{aligned}
$$


hence the differential equation is

$$
\left(1+P^{\prime 2}\right)\left\{p^{2}\left(p^{\prime} q^{\prime \prime}-p^{\prime \prime} q^{\prime}\right)+2 p p^{\prime 2} q^{\prime}\right\}-p^{2} p^{\prime 2} q^{\prime} P^{\prime} P^{\prime \prime}+p^{3} q^{\prime 3}=0 ;
$$

this has an integral

or say

$$
\frac{\left(1+P^{\prime 2}\right) p^{\prime 2}}{p^{4} q^{\prime 2}}+\frac{1}{p^{2}}=\frac{1}{C^{2}}
$$

where

$$
C^{2} s^{\prime 2}=p^{4} q^{\prime 2}
$$

$$
s^{\prime 2}=\left(1+P^{\prime 2}\right) p^{\prime 2}+p^{2} q^{\prime 2} .
$$

Writing here $\rho, \psi$ for $p, q$, where $\rho$ is the distance of the point from the axis, and $\psi$ is the longitude reckoned from an arbitrary meridian, then the equation is

$$
C d s=\rho^{2} d \psi,
$$

which is the equation given by Legendre, Théorie des fonctions elliptiques, t. I. p. 361. This may also be written

$$
\frac{C}{\rho}=\cos \gamma
$$

if $\gamma$ be the inclination of the geodesic line to the parallel of latitude.]

Geodesics on a Quadric Surface.

12. In the case of a quadric surface $\frac{x^{2}}{a}+\frac{y^{2}}{b}+\frac{z^{2}}{c}=1$, writing for shortness $\alpha, \beta, \gamma=b-c, c-a, a-b$, we may express the coordinates $x, y, z$ in terms of two parameters $p, q$ as follows:

$$
\begin{aligned}
& -\beta \gamma x^{2}=a(a+p)(a+q), \\
& -\gamma \alpha y^{2}=b(b+p)(b+q), \\
& -\alpha \beta z^{2}=c(c+p)(c+q),
\end{aligned}
$$

where, in fact, $p=$ const., $q=$ const. are the equations of the two sets of curves of curvature respectively. Writing moreover

we have

$$
P=\frac{p}{(a+p)(b+p)(c+p)}, \quad Q=\frac{q}{(a+q)(b+q)(c+q)},
$$

that is

$$
d s^{2}=\frac{1}{4}(p-q)\left(P d p^{2}-Q d q^{2}\right),
$$

$$
E, F, G=\frac{1}{4}(p-q) P, 0, \frac{1}{4}(q-p) Q ;
$$

and the geodesic equation becomes

$$
\begin{aligned}
& P p^{\prime}\left\{P p^{\prime 2}-2 Q p^{\prime} q^{\prime}+\left(Q+\overline{q-p} Q^{\prime}\right) q^{\prime 2}\right\} \\
+ & Q q^{\prime}\left\{\left(P+\overline{p-q} P^{\prime}\right) p^{\prime 2}-2 P p^{\prime} q^{\prime}+Q q^{\prime 2}\right\} \\
-\quad & 2(p-q) P Q\left(p^{\prime} q^{\prime \prime}-p^{\prime \prime} q^{\prime}\right)=0,
\end{aligned}
$$


where $P^{\prime}, Q^{\prime}$ stand for $d_{p} P$ and $d_{q} Q$ respectively; viz. this is

$$
\begin{aligned}
& p^{\prime 3 .} \cdot P^{2} \\
+ & p^{\prime 2} q^{\prime} \cdot-P Q+(p-q) P^{\prime} Q \\
+ & p^{\prime} q^{\prime 2} \cdot-P Q+(q-p) P Q^{\prime} \\
+ & q^{\prime 3} \cdot \quad Q^{2} \\
- & 2(p-q) P Q\left(p^{\prime} q^{\prime \prime}-p^{\prime \prime} q^{\prime}\right)=0 .
\end{aligned}
$$

13. This has a first integral,

$$
p^{\prime} \sqrt{\left(\frac{P}{\theta+p}\right)+q^{\prime}} \sqrt{\left(\frac{Q}{\theta+q}\right)=0}
$$

where $\theta$ is the constant of integration; or say this is

$$
\theta\left(p^{\prime 2} P-q^{\prime 2} Q\right)+p^{\prime 2} q P-q^{\prime 2} p Q=0 ;
$$

viz. differentiating logarithmically, this gives

$$
\frac{2 p^{\prime} p^{\prime \prime} P-2 q^{\prime} q^{\prime \prime} Q+p^{\prime 3} P^{\prime}-q^{\prime 3} Q^{\prime}}{p^{\prime 2} P-q^{\prime 2} Q}=\frac{2 p^{\prime} p^{\prime \prime} q P-2 q^{\prime} q^{\prime \prime} p Q+p^{\prime 2}\left(q p^{\prime} P^{\prime}+q^{\prime} P\right)-q^{\prime 2}\left(p q^{\prime} Q^{\prime}+p^{\prime} Q\right)}{p^{\prime 2} q P-q^{\prime 2} p Q},
$$

which, multiplying out the denominators, is in fact the foregoing geodesic equation. To verify, consider first the part involving $p^{\prime \prime}, q^{\prime \prime}$ : this is

which is

$$
\left(2 p^{\prime} p^{\prime \prime} P-2 q^{\prime} q^{\prime \prime} Q\right)\left(p^{\prime 2} q P-q^{\prime 2} p Q\right)-\left(2 p^{\prime} p^{\prime \prime} q P-2 q^{\prime} q^{\prime \prime} p Q\right)\left(p^{\prime 2} P-q^{\prime 2} Q\right),
$$

that is

$$
=2 p^{\prime} p^{\prime \prime} P \cdot q^{\prime 2} Q(q-p)-2 q^{\prime} q^{\prime \prime} Q \cdot p^{\prime 2} P(q-p),
$$

or say

$$
=2(q-p) P Q p^{\prime} q^{\prime}\left(p^{\prime \prime} q^{\prime}-p^{\prime} q^{\prime \prime}\right)
$$

$$
=2(p-q) P Q p^{\prime} q^{\prime}\left(p^{\prime} q^{\prime \prime}-p^{\prime \prime} q^{\prime}\right) \text {. }
$$

We have next the part

$$
\left(p^{\prime 3} P^{\prime}-q^{\prime 3} Q^{\prime}\right)\left(p^{\prime 2} q P-q^{\prime 2} p Q\right)-\left\{p^{\prime 2}\left(q p^{\prime} P^{\prime}+q^{\prime} P\right)+q^{\prime 2}\left(p q^{\prime} Q^{\prime}+p^{\prime \prime} Q\right)\right\}\left(p^{\prime 2} P-q^{\prime 2} Q\right),
$$

which is readily found to be

$$
=-p^{\prime} q^{\prime}\left\{p^{\prime 3} P^{2}+p^{\prime 2} q^{\prime}\left(-P Q+\overline{p-q} P^{\prime} Q\right)+p^{\prime} q^{\prime 2}\left(-P Q+\overline{q-p} P Q^{\prime}\right)+q^{\prime 3} Q^{2}\right\}
$$

and the equation is thus verified.

14. We have consequently

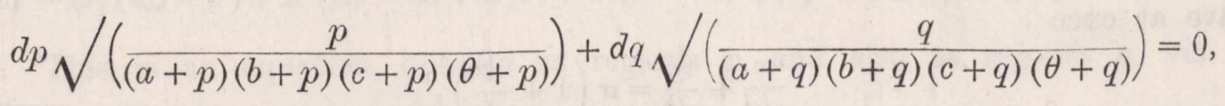

involving the arbitrary constant $\theta$ as the differential equation of the first order of the geodesic lines on the quadric surface $\frac{x^{2}}{a}+\frac{y^{2}}{b}+\frac{z^{2}}{c}=1$ : the geodesic lines in question 
all touch the curve of curvature determined by the parameter $\theta$, that is the curve which is the intersection of the surface by the confocal surface

$$
\frac{x^{2}}{a+\theta}+\frac{y^{2}}{b+\theta}+\frac{z^{2}}{c+\theta}=1 \text {. }
$$

15. In the particular case $\theta=\infty$, the equation becomes

that is

$$
P d p^{2}-Q d q^{2}=0
$$

$$
\frac{p d p^{2}}{(a+p)(b+p)(c+p)}-\frac{q d q^{2}}{(a+q)(b+q)(c+q)}=0,
$$

which is the differential equation of the circular curves on the surface.

16. The signification of the case $\theta=0$ is not at first sight so obvious. Supposing that $\theta$ is first indefinitely small, and writing the equation in the form

$$
\frac{x^{2}}{a}+\frac{y^{2}}{b}+\frac{z^{2}}{c}-1-\theta\left(\frac{x^{2}}{a^{2}}+\frac{y^{2}}{b^{2}}+\frac{z^{2}}{c^{2}}\right)+\& c .=0,
$$

we have the series of geodesics touching the (imaginary) curve of curvature, the intersection of the surface by the imaginary cone $\frac{x^{2}}{a^{2}}+\frac{y^{2}}{b^{2}}+\frac{z^{2}}{c^{2}}=0$. These are, in fact, the right lines on the surface: I apprehend that the intersection in question is not a proper envelope, but is the locus of nodes of the geodesics, viz. each geodesic is to be considered as a pair of lines belonging to the two sets: I do not, however, quite understand this.

17. I say that the geodesics in question are- the right lines on the surface; viz. writing in the differential equation $\theta=0$, it is to be shown that the differential equation of the right lines is

$$
\frac{d p}{\sqrt{(a+p)(b+p)(c+p)}}+\frac{d q}{\sqrt{(a+q)(b+q)(c+q)}}=0
$$

or what is the same thing, that the integral of this equation represents the right lines on the surface.

Writing the equation of the surface in the form

$$
\frac{x^{2}}{a}+\frac{y^{2}}{b}=1-\frac{z^{2}}{c}
$$

we have at once

$$
\begin{aligned}
& \frac{x}{\sqrt{ } a}+\frac{i y}{\sqrt{ } b}=\sigma\left(1+\frac{z}{\sqrt{ } c}\right), \\
& \frac{x}{\sqrt{ } a}-\frac{i y}{\sqrt{ } b}=\frac{1}{\sigma}\left(1-\frac{z}{\sqrt{ } c}\right),
\end{aligned}
$$


( $\sigma$ an arbitrary parameter) as the equations of a right line on the surface; viz. considering $x, y, z$ as denoting the foregoing functions of $p$ and $q$, these two equations are forms of a single relation between $p, q, \sigma$, which relation expresses that the point $(p, q)$ is situate on the right line determined by the parameter $\sigma$. We may from this integral equation deduce without difficulty the foregoing differential equation; viz. we have

or multiplying these equations,

$$
\begin{aligned}
& \frac{d x}{\sqrt{ } a}+\frac{i d y}{\sqrt{ } b}=\sigma \frac{d z}{\sqrt{ } c}, \\
& \frac{d x}{\sqrt{ } a}-\frac{i d y}{\sqrt{ } b}=-\frac{1}{\sigma} \frac{d z}{\sqrt{ } c},
\end{aligned}
$$

$$
\frac{d x^{2}}{a}+\frac{d y^{2}}{b}+\frac{d z^{2}}{c}=0
$$

and substituting herein for $d x, d y, d z$ their values in terms of $d p$ and $d q$, we find the required equation

$$
\frac{d p^{2}}{(a+p)(b+p)(c+p)}-\frac{d q^{2}}{(a+q)(b+q)(c+q)}=0 .
$$

18. I return to the integral equation involving $\sigma$ : we have to rationalise this equation, that is, obtain from it an equation containing $x^{2}, y^{2}, z^{2}$, and then substituting for these their values in terms of $p, q$, we have the required relation between $p, q, \sigma$. We at once obtain

$$
\left\{2\left(\frac{x^{2}}{a}-\frac{y^{2}}{b}\right)-\left(\sigma^{2}+\frac{1}{\sigma^{2}}\right)\left(1+\frac{z^{2}}{c}\right)\right\}^{2}-4\left(\sigma^{2}-\frac{1}{\sigma^{2}}\right)^{2} \frac{z^{2}}{c}=0 ;
$$

or if for greater convenience we introduce in place of $\sigma$ a new parameter $\phi$, determined by the equation $\sigma^{2}+\frac{1}{\sigma^{2}}=\frac{2 \phi}{\gamma}$, the equation is

$$
\left\{\gamma\left(\frac{x^{2}}{a}-\frac{y^{2}}{b}\right)-\phi\left(1+\frac{z^{2}}{c}\right)\right\}^{2}-4\left(\phi^{2}-\gamma^{2}\right) \frac{z^{2}}{c}=0 .
$$

Writing for shortness $p+q=X, p q=Y$, we have

$$
-\beta \gamma \frac{x^{2}}{a}=a^{2}+a X+Y, \quad-\gamma \alpha \frac{y^{2}}{b}=b^{2}+b X+Y, \quad-\alpha \beta \frac{z^{2}}{c}=c^{2}+c X+Y ;
$$

and substituting these values, the equation becomes

$$
\left\{\beta\left(b^{2}+b X+Y\right)-a\left(a^{2}+a X+Y\right)-\phi \gamma\left(\alpha \beta-c^{2}-c X-Y\right)\right\}^{2}+4 \alpha \beta\left(\phi^{2}-\gamma^{2}\right)\left(c^{2}+c X+Y\right)=0,
$$

or, what is the same thing,

$\left\{\beta b^{2}-\alpha a^{2}-\phi \gamma\left(\alpha \beta-c^{2}\right)+X(\beta b-\alpha a+\phi \gamma c)+Y(\beta-\alpha+\phi \gamma)\right\}^{2}+4 \alpha \beta\left(\phi^{2}-\gamma^{2}\right)\left(c^{2}+c X+Y\right)=0$.

19. This is an equation, quadric as regards $p$, and also as regards $q$, viz. it is of the form

$$
\begin{gathered}
\left(\mathrm{a}+2 \mathrm{~h} p+\mathrm{g} p^{2}\right) \\
+2 q\left(\mathrm{~h}+2 \mathrm{~b} p+\mathrm{f} p^{2}\right) \\
+q^{2}\left(\mathrm{~g}+2 \mathrm{f} p+c p^{2}\right)=0,
\end{gathered}
$$


and it leads to a differential equation

where

$$
\frac{d p}{\sqrt{ } P}+\frac{d q}{\sqrt{ } Q}=0
$$

$$
\begin{aligned}
& P=\left(\mathrm{h}+2 \mathrm{~b} p+\mathrm{f} p^{2}\right)^{2}-\left(\mathrm{a}+2 \mathrm{~h} p+\mathrm{g} p^{2}\right)\left(\mathrm{g}+2 \mathrm{f} p+\mathrm{c} p^{2}\right) \\
& Q=\left(\mathrm{h}+2 \mathrm{~b} q+\mathrm{f} q^{2}\right)^{2}-\left(\mathrm{a}+2 \mathrm{~h} q+\mathrm{g} q^{2}\right)\left(\mathrm{g}+2 \mathrm{f} q+\mathrm{c} q^{2}\right)
\end{aligned}
$$

and upon effecting the calculation, it is found that we have

$$
\begin{aligned}
& P=-8 \alpha^{2} \beta^{2}\left(\phi^{2}-\gamma^{2}\right)(a+b-2 c-\phi)(a+p)(b+p)(c+p) \\
& Q=-8 \alpha^{2} \beta^{2}\left(\phi^{2}-\gamma^{2}\right)(a+b-2 c-\phi)(a+q)(b+q)(c+q)
\end{aligned}
$$

viz. $P, Q$ are the same multiples of $(a+p)(b+p)(c+p),(a+q)(b+q)(c+q)$ respectively; so that, omitting the common factor, or taking $P, Q$ to represent the lastmentioned functions respectively, we have

$$
\frac{d p}{\sqrt{ } P}+\frac{d q}{\sqrt{ } Q}=0
$$

and since the parameter $\phi$ has disappeared, we see that the original equation involving $\phi$ is the general integral of this differential equation; viz. that the differential equation belongs to the right lines on the surface.

20. The form of the integral equation may be simplified by introducing instead of $\phi$ a new parameter $K$, connected with it by the equation

$$
K=\frac{\beta b-\alpha a+\phi c}{\beta-\alpha+\phi}, \text { or } \phi=\frac{(\beta-\alpha) K-\beta b+\alpha a}{c-K},
$$

viz. we thence deduce

$$
\begin{array}{rlrl}
\beta-\alpha+\phi & =-2 \alpha \beta & & (\div) \\
\beta b-\alpha a+\phi c & =-2 \alpha \beta K & & (\div), \\
\beta b^{2}-\alpha a^{2}-\phi\left(\alpha \beta-c^{2}\right) & =2 \alpha \beta(a c+b c-a b+2 c K) & & (\div) \\
\phi+\gamma & =2 \beta(K-b) & & (\div) \\
\phi-\gamma & =-2 \alpha(K-a) &
\end{array}
$$

where the sign $(\div)$ is used to signify that the functions preceding it have to be divided by a denominator which in fact is $=c-K$. The equation thus becomes

$$
(a c+b c-a b-2 c K-K X-Y)^{2}-4(K-a)(K-b)\left(c^{2}+c X+Y\right)=0 ;
$$

and if we moreover write

$$
\nu, \mu, \lambda=a b c, b c+c a+a b, a+b+c,
$$

and instead of $K$ introduce the parameter $C,=\lambda-K$, the equation becomes

$$
\left\{-\mu-2 c^{2}+2 c C-(\lambda-C) X-Y\right\}^{2}+(b+c-C)(c+a-C)\left(c^{2}+c X+Y\right)=0 ;
$$


or expanding and reducing, this is

or say

$$
\begin{aligned}
& \{Y+(\lambda-C) X\}^{2} \\
& \quad+Y\left(-2 \mu+4 \lambda C-4 C^{2}\right) \\
& \quad+X(2 \mu \lambda-4 \nu-2 \mu C) \\
& \quad+\mu^{2}-4 \nu C=0
\end{aligned}
$$

$$
\begin{aligned}
& \mu^{2}-4 \nu C \\
+ & (2 \mu \lambda-4 \nu-2 \mu C)(p+q) \\
+ & \left(-2 \mu+4 \lambda C-4 C^{2}\right) p q \\
+ & (\lambda-C)^{2}\left(p^{2}+q^{2}+2 p q\right) \\
+ & 2(\lambda-C) p q(p+q) \\
+ & p^{2} q^{2} \quad=0
\end{aligned}
$$

viz. this, containing the constant $C$, is the general integral of the differential equation

where

$$
\frac{d p}{\sqrt{ } P}+\frac{d q}{\sqrt{ } Q}=0
$$

$$
\begin{array}{ll}
P=(a+p)(b+p)(c+p), & =\nu+\mu p+\lambda p^{2}+p^{3}, \\
Q=(a+q)(b+q)(c+q), & =\nu+\mu q+\lambda q^{2}+q^{3} .
\end{array}
$$

21. The constant $C$ is connected with the parameter $\sigma$, which originally served to determine the right line, by the equation

or, what is the same thing,

$$
\sigma+\frac{1}{\sigma}=\frac{2}{\gamma} \frac{(\beta-\alpha)(\lambda-C)-\beta b+\alpha a}{c-(\lambda-C)},
$$

$$
\sigma+\frac{1}{\sigma}=\frac{2}{b-c} \frac{2 c^{2}-a^{2}-b^{2}-C(2 c-a-b)}{C-a-b} .
$$

Reverting to the equation between $p, q, \phi$, I remark that if $\phi$ be therein considered as variable, we have the differential equation

$$
\sqrt{ } Q d p+\sqrt{ } P d q+\sqrt{ } \Phi d \phi=0
$$

where $P, Q$ have the foregoing values

$$
P=-8 \alpha^{2} \beta^{2}\left(\phi^{2}-\gamma^{2}\right)(a+b-c-\phi)(a+p)(b+p)(c+p), \quad Q=\& c . ;
$$

and where, if the integral equation be written in the form

$$
L+2 M \phi+N \phi^{2}=0,
$$

then we have $\Phi=M^{2}-N L$, viz. we thus find

$$
\Phi=16 \alpha^{2} \beta^{2}(a+p)(b+p)(c+p)(a+q)(b+q)(c+q) .
$$

C. VIII. 
22. Changing the notation, and writing

the equation is

$$
\begin{aligned}
& P=(a+p)(b+p)(c+p), \\
& Q=(a+q)(b+q)(c+q), \\
& \Phi=\left(\phi^{2}-\gamma^{2}\right)(a+b-2 c-\phi),
\end{aligned}
$$

$$
\frac{d p}{\sqrt{ } P}+\frac{d q}{\sqrt{ } Q}+\frac{\sqrt{ } 2 d \phi}{\sqrt{ } \Phi}=0
$$

or if, instead of $\phi$, we introduce the original parameter $\sigma$, then, observing that

$$
\frac{2 d \sigma}{\sigma}=\frac{d \phi}{\sqrt{\phi^{2}-\gamma^{2}}},
$$

we at once find

$$
\frac{d p}{\sqrt{ } P}+\frac{d q}{\sqrt{ } Q}+\frac{4 d \sigma}{\sqrt{ } \Sigma}=0
$$

where

$$
\Sigma=\gamma\left(1+\sigma^{4}\right)-2(a+b-2 c) \sigma^{2}
$$

or, what is the same thing,

$$
\Sigma=a\left(\sigma^{2}-1\right)^{2}-b\left(\sigma^{2}+1\right)^{2}+c .4 \sigma^{2}
$$

viz. passing from a point $(p, q)$ on the line $\sigma$ to a consecutive point $(p+d p, q+d q)$ on the line $\sigma+d \sigma$, the above is the relation between the variations $d p, d q$, $d \sigma$. If $\tau$ be the parameter of the other line through the same point, then we have in like manner, say

$$
\frac{d p}{\sqrt{ } P}-\frac{d q}{\sqrt{ } Q}+\frac{4 d \tau}{\sqrt{ } \mathrm{T}}=0
$$

(viz. one of the radicals $\sqrt{ } P, \sqrt{ } Q$ must present itself with a reversed sign): and we thus have $d p, d q$ each expressed in terms of $d \sigma, d \tau$; viz. we have the increments $d p, d q$ when a point passes from $(\sigma, \tau)$ to $(\sigma+d \sigma, \tau+d \tau)$. These results will be presently obtained in a more simple manner.

Formula where the position of a Point on the Surface is determined by means of the two Lines through the Point.

23. We may determine the position of a point by means of the parameters $\sigma, \tau$ of the two lines through the point. The equations of these are

$$
\begin{aligned}
& \frac{x}{\sqrt{ } a}+\frac{i y}{\sqrt{ } b}=\sigma\left(1+\frac{z}{\sqrt{ } c}\right), \quad \frac{x}{\sqrt{a}}+\frac{i y}{\sqrt{ } b}=\tau\left(1-\frac{z}{\sqrt{ }}\right), \\
& \frac{x}{\sqrt{ } a}-\frac{i y}{\sqrt{ } b}=\frac{1}{\sigma}\left(1-\frac{z}{\sqrt{ } c}\right), \quad \frac{x}{\sqrt{ } a}-\frac{i y}{\sqrt{ } b}=\frac{1}{\tau}\left(1+\frac{z}{\sqrt{ } c}\right),
\end{aligned}
$$


and from these equations we deduce

We have thence

$$
\frac{x}{\sqrt{ } a}=\frac{\sigma \tau+1}{\tau+\sigma}, \quad \frac{i y}{\sqrt{ } b}=\frac{\sigma \tau-1}{\tau+\sigma}, \quad \frac{z}{\sqrt{ } c}=\frac{\tau-\sigma}{\tau+\sigma} .
$$

$$
\begin{aligned}
& \frac{d x}{\sqrt{ } a}=\left(\tau^{2}-1\right) d \sigma+\left(\sigma^{2}-1\right) d \tau \\
& i d y=\left(\tau^{2}+1\right) d \sigma+\left(\sigma^{2}+1\right) d \tau \quad \\
& \left.\frac{\sqrt{\sqrt{b}}}{\div}\right), \\
& \frac{d z}{\sqrt{ } c}=-2 \tau d \sigma+\quad 2 \sigma d \tau \quad(\div),
\end{aligned}
$$

where denom. $=(\tau+\sigma)^{2}$ : regarding $\sigma, \tau$ as the parameters in place of $p$, $q$, these show the values of the first differential coefficients $a, a^{\prime} ; b, b^{\prime} ; c, c^{\prime}$. We deduce

$$
A=-2 i \sqrt{b c}(\sigma \tau+1) \div, \quad B=-2 i \sqrt{c a}(\sigma \tau-1) \div, C=-2 i \sqrt{b a}(\tau-\sigma) \div,
$$

where denom. $=(\tau+\sigma)^{3}$. We have, moreover,

$$
\begin{aligned}
& \frac{d^{2} x}{\sqrt{ } a}=-2\left(\tau^{2}-1\right) d \sigma^{2}+2(\sigma \tau+1) 2 d \sigma d \tau-2\left(\sigma^{2}-1\right) d \tau^{2} \quad(\div), \\
& 2 d^{2} y=-2\left(\tau^{2}+1\right) d \sigma^{2}+2(\sigma \tau-1) 2 d \sigma d \tau-2\left(\sigma^{2}+1\right) d \tau^{2} \quad(-), \\
& \sqrt{ } b=4 \pi d \sigma^{2}+2(\tau-\sigma) 2 d \sigma d \tau+\quad 4 \sigma d \tau^{2} \quad(\div), \\
& \frac{d^{2} z}{\sqrt{ } c}=+\quad 4 \tau \quad
\end{aligned}
$$

where denom. $=(\tau+\sigma)^{3}$ : giving $\alpha, \alpha^{\prime}, \alpha^{\prime \prime} ; \beta, \beta^{\prime}, \beta^{\prime \prime} ; \gamma, \gamma^{\prime}, \gamma^{\prime \prime}$. We deduce as the numerators of $E^{\prime}(=A \alpha+B \beta+C \gamma)$ and $G^{\prime}\left(=A \alpha^{\prime \prime}+B \beta^{\prime \prime}+C \gamma^{\prime \prime}\right)$,

and

$$
4 i \sqrt{a b c}\left\{(\sigma \tau+1)\left(\tau^{2}-1\right)-(\sigma \tau-1)\left(\tau^{2}+1\right)-2 \tau(\tau-\sigma)\right\},=0,
$$

$$
4 i \sqrt{a b c}\left\{(\sigma \tau+1)\left(\sigma^{2}-1\right)-(\sigma \tau-1)\left(\sigma^{2}+1\right)+2 \sigma(\tau-\sigma)\right\},=0
$$

that is, $E^{\prime}=0$ and $G^{\prime}=0$; or the differential equation of the chief lines is $d \sigma d \tau=0$, which is right. The value of $F^{\prime \prime}\left(=A \alpha^{\prime}+B \beta^{\prime}+C \gamma^{\prime}\right)$ is hardly required, but it is readily found to

$$
=4 i \sqrt{a b c}\left\{-(\sigma \tau+1)^{2}+(\sigma \tau-1)^{2}-(\tau-\sigma)\right\}^{2} \div(\tau+\sigma)^{6},
$$

or since the term in \{\} is $=-(\tau+\sigma)^{2}$, we have

$$
F^{\prime}=\frac{-4 i \sqrt{a b c}}{(\tau+\sigma)^{4}}
$$

24. The values of $E, F, G\left(d s^{2}=E d \sigma^{2}+2 F d \sigma d \tau+G d \tau^{2}\right)$ are

$$
\begin{aligned}
& E=a\left(\tau^{2}-1\right)^{2} \quad-b\left(\tau^{2}+1\right)^{2} \quad+c .4 \tau^{2} \quad(\div) \text {, } \\
& F=a\left(\tau^{2}-1\right)\left(\sigma^{2}-1\right)-b\left(\tau^{2}+1\right)\left(\sigma^{2}+1\right)-c .4 \tau \sigma \quad(\div) \text {, } \\
& G=a\left(\sigma^{2}-1\right)^{2} \quad-b\left(\sigma^{2}+1\right)^{2} \quad+c .4 \sigma^{2} \quad(\div),
\end{aligned}
$$

where denom. $=(\tau+\sigma)^{4}$. 
We have, it is clear, $\left(E_{1}=d_{\sigma} E, E_{2}=d_{\tau} E\right.$, \&c.)

$$
E_{1}=-\frac{4}{\sigma+\tau} E, \quad G_{2}=-\frac{4}{\sigma+\tau} G
$$

Hence the condition $F G_{2}-2 F_{2} G+G G_{1}=0$, in order that $\sigma=$ const. may be a geodesic, reduces itself to

$$
-\frac{4}{\sigma+\tau} F-2 F_{2}+G_{1}=0
$$

and similarly, the condition $-2 E F_{1}+E_{1} F+E E_{2}=0$, in order that $\tau=$ const. may be a geodesic, reduces itself to

$$
-\frac{4}{\sigma+\tau} F-2 F_{1}+E_{2}=0
$$

We have at once

$$
\begin{array}{llll}
E_{2}=4 a\left(\tau^{2}-1\right)(\tau \sigma+1) & -4 b\left(\tau^{2}+1\right)(\tau \sigma-1) & +4 c .2 \tau(\sigma-\tau) & (\div), \\
G_{1}=4 a\left(\sigma^{2}-1\right)(\tau \sigma+1) & -4 b\left(\sigma^{2}+1\right)(\tau \sigma-1) & -4 c .2 \sigma(\sigma-\tau) & (\div), \\
F_{1}=2 a\left(\tau^{2}-1\right)\left(\tau \sigma-\sigma^{2}+2\right)-2 b\left(\tau^{2}+1\right)\left(\tau \sigma-\sigma^{2}-2\right)-2 c .2 \tau(\tau-3 \sigma) & (\div), \\
F_{2}=2 a\left(\sigma^{2}-1\right)\left(\tau \sigma-\tau^{2}+2\right)-2 b\left(\sigma^{2}+1\right)\left(\tau \sigma-\tau^{2}-2\right)-2 c .2 \sigma(\sigma-3 \tau) & (\div),
\end{array}
$$

where denom. $=(\sigma+\tau)^{5}$; and substituting these values, the conditions are verified: we thus again see $\dot{a}$ posteriori that the right lines $\sigma=$ const. and $\tau=$ const. are geodesics.

25. The last-mentioned values of $E, G$ are $E=\mathrm{T} \div(\tau+\sigma)^{4}, G=\Sigma \div(\tau+\sigma)^{4}$; and writing for a moment

$$
A=a\left(\tau^{2}-1\right)\left(\sigma^{2}-1\right)-b\left(\tau^{2}+1\right)\left(\sigma^{2}+1\right)-c .4 \sigma \tau,
$$

we have $F:=A \div(\tau+\sigma)^{4}$, the value of $d s^{2}$ is thus

$$
=\mathrm{T} d \sigma^{2}+2 A d \sigma d \tau+\Sigma d \tau^{2} \div(\tau+\sigma)^{4},
$$

which should be

where, as before,

$$
=\frac{1}{4}(p-q)\left(p \frac{d p^{2}}{P}-q \frac{d q^{2}}{Q}\right)
$$

$$
P, Q=(a+p)(b+p)(c+p), \quad(a+q)(b+q)(c+q),
$$

respectively. We have already found

or, what is the same thing,

$$
\begin{aligned}
& \frac{d p}{\sqrt{ } P}+\frac{d q}{\sqrt{ } Q}+\frac{4 d \sigma}{\sqrt{ } \Sigma}=0 \\
& \frac{d p}{\sqrt{ } P}-\frac{d q}{\sqrt{ } Q}+\frac{4 d \tau}{\sqrt{ } \mathrm{T}}=0
\end{aligned}
$$

$$
\begin{aligned}
& \frac{d p}{\sqrt{ } P}=-2\left(\frac{d \sigma}{\sqrt{ } \Sigma}+\frac{d \tau}{\sqrt{\mathrm{T}}}\right), \\
& \frac{d q}{\sqrt{ } Q}=-2\left(\frac{d \sigma}{\sqrt{ } \Sigma}-\frac{d \tau}{\sqrt{\mathrm{T}}}\right)
\end{aligned}
$$


and we ought therefore to have identically

that is

$$
(p-q)\left\{p\left(\frac{d \sigma}{\sqrt{ } \Sigma}+\frac{d \tau}{\sqrt{T}}\right)^{2}-q\left(\frac{d \sigma}{\sqrt{ } \Sigma}-\frac{d \tau}{\sqrt{ } \mathrm{T}}\right)^{2}\right\}=\mathrm{T} d \sigma^{2}+2 A d \sigma d \tau+\Sigma d \tau^{2} \div(\tau+\sigma)^{4}
$$

or, what is the same thing,

$$
\begin{aligned}
& (p-q)^{2}=\quad \mathrm{T} \Sigma \div(\tau+\sigma)^{4}, \\
& p^{2}-q^{2}=A \sqrt{\mathrm{T} \Sigma} \div(\tau+\sigma)^{4}
\end{aligned}
$$

$$
\begin{array}{ll}
(p-q)^{2}= & \mathrm{T} \Sigma \div(\tau+\sigma)^{4}, \\
p+q= & A \div(\tau+\sigma)^{2}
\end{array}
$$

which are easily verified.

26. In fact, the equation

$$
\frac{x^{2}}{a+u}+\frac{y^{2}}{b+u}+\frac{z^{2}}{c+u}=1
$$

gives for $u$ a quadric equation, the roots of which are $u=p, u=q$; that is, we have

$$
\begin{aligned}
& p+q=x^{2}+y^{2}+z^{2}-a-b-c \\
& p q=-(b+c) x^{2}-(c+a) y^{2}-(a+b) z^{2}+b c+c a+a b
\end{aligned}
$$

and substituting herein for $x^{2}, y^{2}, z^{2}$ their values in terms of $\sigma, \tau$, we find

$$
\begin{array}{ll}
p+q=a\left(\sigma^{2}-1\right)\left(\tau^{2}-1\right)-b\left(\sigma^{2}+1\right)\left(\tau^{2}+1\right)-4 c \tau \sigma & \div(\tau+\sigma)^{2}, \\
p q=b c(\sigma \tau+1)^{2}-c a(\sigma \tau-1)^{2}+a b(\sigma-\tau)^{2} & \div(\tau+\sigma)^{4}
\end{array}
$$

the first of which is, in fact, $p+q=A \div(\tau+\sigma)^{2}$. And from the two equations, forming the combination $(p+q)^{2}-4 p q$, we at once obtain the other equation

$$
(p-q)^{2}=\Sigma \mathrm{T} \div(\tau+\sigma)^{4} \text {. }
$$

27. The most ready way of obtaining the relations between the differentials of $p, q, \sigma, \tau$, is from the foregoing expressions of $p+q, p q$. Writing for a moment $p+q=A \div(\sigma+\tau)^{2}, p q=B \div(\sigma+\tau)^{2}$, we have $p^{2}(\sigma+\tau)^{2}-A p+B=0, q^{2}(\sigma+\tau)^{2}-A q+B=0$; viz. the first of these equations is

$$
\begin{aligned}
p^{2}(\sigma+\tau)^{2}-p\left[a\left(\sigma^{2}-1\right)\left(\tau^{2}-1\right)-\right. & \left.b\left(\sigma^{2}+1\right)\left(\tau^{2}+1\right)-4 c \sigma \tau\right] \\
& +b c(\sigma \tau+1)^{2}-c a(\sigma \tau-1)^{2}+a b(\sigma-\tau)^{2}=0
\end{aligned}
$$

which is quadric in $p, \sigma, \tau$. The negative discriminants in regard to these variables respectively are $\Sigma \mathrm{T}, 4 P \mathrm{~T}, 4 P \Sigma$ respectively, and we have thus the equation

and the like equation for $d q$.

$$
\frac{d p}{\sqrt{ } P}+2\left(\frac{d \sigma}{\sqrt{ } \Sigma}+\frac{d \tau}{\sqrt{ } \mathrm{T}}\right)=0
$$

28. In the first integral of the geodesic lines, introducing $\sigma, \tau$ instead of $p, q$, the equation becomes

$$
\sqrt{\left(\frac{p}{p+\theta}\right)}\left(\frac{d \sigma}{\sqrt{\Sigma}}+\frac{d \tau}{\sqrt{\mathrm{T}}}\right)-\sqrt{\left(\frac{q}{q+\theta}\right)}\left(\frac{d \sigma}{\sqrt{ } \Sigma}-\frac{d \tau}{\sqrt{\mathrm{T}}}\right)=0
$$


or, what is the same thing,

that is,

$$
p(q+\theta)\left(\frac{d \sigma}{\sqrt{\Sigma}}+\frac{d \tau}{\sqrt{T}}\right)^{2}-q(p+\theta)\left(\frac{d \sigma}{\sqrt{\Sigma}}-\frac{d \tau}{\sqrt{ } \mathrm{T}}\right)^{2}=0
$$

$$
(p-q) \theta\left(\frac{d \sigma^{2}}{\Sigma}+\frac{d \tau^{2}}{\mathrm{~T}}\right)+2[2 p q+\theta(p+q)] \frac{d \sigma d \tau}{\sqrt{\Sigma T}}=0
$$

or substituting herein for $p-q, p q, p+q$ the values $\sqrt{\Sigma \mathrm{T}}, B, A$, each divided by $(\sigma+\tau)^{2}$, this is

$$
\theta\left(\mathrm{T} d \sigma^{2}+\Sigma d \tau^{2}\right)+2(2 B+\theta A) d \sigma d \tau=0
$$

or say

$$
\theta\left(\mathrm{T} d \sigma^{2}+2 A d \sigma d \tau+\Sigma d \tau^{2}\right)+4 B d \sigma d \tau=0
$$

viz. writing herein $\theta=0$, the equation is $d \sigma d \tau=0$, giving the right lines on the surface; and writing $\theta=\infty$, it is $\mathrm{T} d \sigma^{2}+2 A d \sigma d \tau+\Sigma d \tau^{2}=0$, giving the circular lines.

29. The equation $d s^{2}=\mathrm{T} d \sigma^{2}+2 A d \sigma d \tau+\Sigma d \tau^{2} \div(\tau+\sigma)^{4}$ shows that the right lines $\sigma, \sigma+d \sigma, \tau, \tau+d \tau$ form on the surface an indefinitely small parallelogram, the sides whereof are $\sqrt{ } \mathrm{T} d \sigma \div(\tau+\sigma)^{2}$ and $\sqrt{ } \Sigma d \tau \div(\tau+\sigma)^{2}$, viz. the ratio of the coefficients of $d \sigma, d \tau$ is of the form function $\sigma \div$ function $\tau$; and it thus appears that it is possible to draw on the surface the two sets of right lines, the lines of each set being at such intervals that the surface is divided into parallelograms, the sides of which have to each other any given ratio (the angles being variable); viz. if this ratio be as $m: 1$, then, to determine the relation between $\sigma, \tau$, we must have $\sqrt{ } \mathrm{T} d \sigma= \pm m \sqrt{ } \Sigma d \tau$, or what is the same thing, $\frac{d \sigma}{\sqrt{ } \Sigma}= \pm m \frac{d \tau}{\sqrt{T}}$. In particular, if $m=1$, the parallelograms will be rhombs; and we must then have

$$
\frac{d \sigma}{\sqrt{\Sigma}}= \pm \frac{d \tau}{\sqrt{T}}
$$

viz. this being in terms of $\sigma, \tau$, the differential equation of the curves of curvature, it appears that the two sets of lines may be taken so as to divide the surface into indefinitely small rhombs, such that, drawing the diagonals of these, we have, the two sets of curves of curvature.

\section{The Ellipsoid and the Skew Hyperboloid.}

30. I have thus far considered a quadric surface in general, the various theorems being applicable as well to the ellipsoid and the hyperboloid of two sheets as to the skew hyperboloid, the right lines being of course imaginary for the first-mentioned surfaces; but I will now consider the ellipsoid and the skew hyperboloid separately.

31. First the ellipsoid. We have here $a, b, c$ all positive, and $I$ assume as usual $a>b>c$. The principal sections are all ellipses, viz. $\frac{x^{2}}{a}+\frac{y^{2}}{b}=1$ is the major- 
mean, or say the minor section, $\frac{y^{2}}{b}+\frac{z^{2}}{c}=1$ the minor-mean, or say the major section, and $\frac{x^{2}}{a}+\frac{z^{2}}{c}=1$ the mean, or umbilicar section. The elliptic coordinates $p, q$ enter into the equations symmetrically, but we distinguish them by taking $p$ to extend from $-c$ to $-b$, and $q$ to extend from $-b$ to $-a$. Thus $p=$ const. denotes the curves of

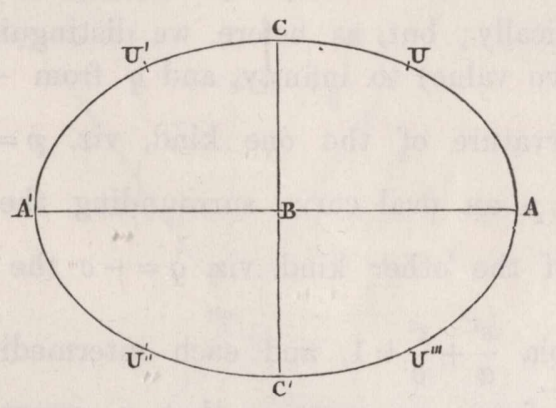

curvature of the one kind; viz. $p=-c$ denotes the major-mean section $\frac{x^{2}}{a}+\frac{y^{2}}{b}=1$, $p=-b$ the portions $U U^{\prime}$ and $U^{\prime \prime} U^{\prime \prime \prime}$ of the umbilicar section; and $q=$ const. denotes the curves of curvature of the other kind, viz. $q=-b$ the remaining portions $U^{\prime} U^{\prime \prime}$ and $U^{\prime \prime \prime} U$ of the umbilicar section, $q=-a$ the minor-mean section $\frac{y^{2}}{b}+\frac{z^{2}}{c}=1$; say $p=$ const. the major-mean curves, and $q=$ const. the minor-mean curves.

32. Hence, in order that the equation

$$
\left.d p \sqrt{\left(\frac{p}{(a+p)(b+p)(c+p)(\theta+p)}\right) \pm d q} \sqrt{\left(\frac{q}{(a+q)(b+q)(c+q)(\theta+q)}\right)}\right)=0
$$

of the geodesic lines may be real \{observing that we have $a+p, b+p=+, c+p$, $p=-$, and $a+q=+, b+q, c+q, q=-$, consequently $p \div(a+p)(b+p)(c+p)=+$, but $q \div(a+q)(b+q)(c+q)=-\}$, we must have $\theta+p, \theta+q$ of opposite signs, that is $\theta+p=+$ and $\theta+q=-$; or $\theta$ included between the limits $a, c$. Or, what is the same thing, $-\theta$ is included between the limits $-c,-b$, say $-\theta$ has a $p$-value; or else between the limits $-b,-a$, say $-\theta$ has a $q$-value. This is conveniently shown

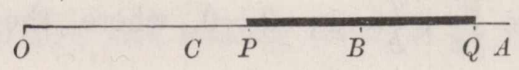

in the annexed diagram of the values of $-p,-q,-\theta$. Hence on the ellipsoid we have two kinds of geodesic lines, each of them touching a real curve of curvature; viz. those which touch a major-mean curve and those which touch a minor-mean curve: the transition case, answering to the value $\theta=b$, is that of the geodesic lines which pass through an umbilicus. I have considered the theory more in detail in my memoir "On the Geodesic Lines of an Ellipsoid," Mem. R. Ast. Soc., t. xxx., pp. 31-53, $1872,[478]$. 
33. Next, for the skew hyperboloid, we have $a$ and $b=+, c=-$, and I assume for convenience $a>b$. Attending to the signs, we still have therefore $a>b>c$. The principal sections are one of them an ellipse, and the other two hyperbolas, viz. the minor section is the ellipse $\frac{x^{2}}{a}+\frac{y^{2}}{b}=1$, the major section is the hyperbola $\frac{y^{2}}{b}+\frac{z^{2}}{c}=1$, and the mean section is the hyperbola $\frac{x^{2}}{a}+\frac{z^{2}}{c}=1$ : there are no umbilici. The elliptic coordinates enter symmetrically; but, as before, we distinguish them, viz. we take $p$ to extend from $-c$ (a positive value) to infinity, and $q$ from $-b$ to $-a$. Thus $p=$ const. denotes the curves of curvature of the one kind, viz. $p=-c$ the ellipse $\frac{x^{2}}{a}+\frac{y^{2}}{b}=1$, and every other value of $p$ an oval curve surrounding the hyperboloid; and $q=$ const. the curves of curvature of the other kind, viz. $q=-c$ the major hyperbola $\frac{y^{2}}{b}+\frac{z^{2}}{c}=1$, $q=-b$ the mean hyperbola $\frac{x^{2}}{a}+\frac{z^{2}}{c}=1$, and each intermediate value gives a curve of curvature of a hyperbolic form: we may say that $p=$ const. determines the oval curves of curvature, and $q=$ const. the hyperbolic curves of curvature.

34. In the equation of the geodesic lines we have $a+p, b+p, c+p, p$ all positive; but $a+q=+, b+q, c+q, q$ each $=-$; hence $p \div(a+p)(b+p)(c+p)=+$, but $q \div(a+q)(b+q)(c+q)=-$; therefore $\theta+p$ and $\theta+q$ must be of opposite signs, or we must have $\theta+p=+$ and $\theta+q=-$; or what is the same thing, $\theta$ may have any value from $-p$ to $-q$, or say $-\theta$ any value from $p$ to $q$; that is, the value of $-\theta$ may be positive and greater than $-c$, positive and less than $-c$, negative and less than $-b$, negative and between $-b$ and $-a$; viz. in the first case $-\theta$ has a $p$-value,

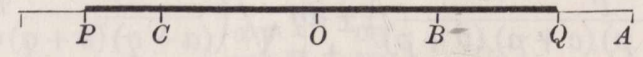

and in the fourth case it has a $q$-value, but in the second and third cases it has neither a $p$ - nor a $q$-value. This is better seen from the diagram. It follows that we have, on the hyperboloid, geodesic lines of four different kinds: those which touch a real curve of curvature, oval or hyperbolic, and those which touch no real curve of curvature, but for which $-\theta$ has a positive value from 0 to $-c$, or a negative value from 0 to $-b$. And there are the transitional cases $-\theta=-c$, where the geodesic touches the ellipse $\frac{x^{2}}{a}+\frac{y^{2}}{b}=1 ; \theta=0$, where the geodesic becomes a right line; and $-\theta=-b$, where the geodesic touches the mean hyperbola $\frac{x^{2}}{a}+\frac{z^{2}}{c}=1$.

35. To explain this more in detail, consider the geodesics which start from a point $M$ of the hyperboloid. To fix the ideas, consider the axis, of $z$ as vertical, and take the point $M$ in the positive octant of the hyperboloid; and let $M 1$ represent the direction of the oval curve of curvature, $M 9$ that of the hyperbolic curve of curvature, M5 that of one of the right lines. 
The geodesic of initial direction $M 1$ touches at $M$ the oval curve of curvature $M 1$, and lies wholly above this curve; it makes an infinity of convolutions round the upper part of the hyperboloid, cutting all the oval curves of curvature for which $p$ has a (positive) value greater than $p_{1}$ (if $p_{1}$ is the value of $p$ corresponding to the ovai curve through $M$ ), and ascending to infinity: or considering the curve as described in the opposite sense, it descends from infinity to touch the oval curve through $M$, after which it again ascends to infinity.

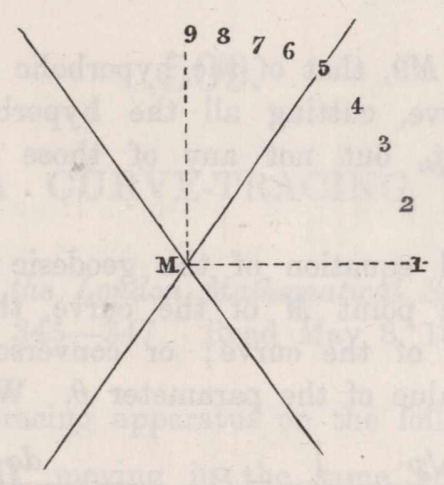

Next, if the initial direction is $M 2$, we have a geodesic of the same kind, only descending below $M$ to touch a certain oval curve having for its parameter $p_{2}\left(p_{2}>-c<p_{1}\right)$.

We come next to a critical direction $M 3$, for which the geodesic descends below $M$ to touch the oval curve of parameter $p_{3}=-c$, that is, the ellipse $\frac{x^{2}}{a}+\frac{y^{2}}{b}=1$. But it is to be observed that, whatever the initial point $M$ may be, the geodesic makes below $M$ an infinity of convolutions round the hyperboloid, so that it does not in fact ever actually touch the ellipse, but has this ellipse for an asymptote. That this is so appears from the consideration that the ellipse, qud plane curve of curvature, is a geodesic; so that, starting from a point of the ellipse in the direction of the ellipse, the geodesic coincides with the ellipse, or, besides the ellipse itself, there is not any geodesic which touches the ellipse.

Next, if the initial direction be $M 4$, the geodesic does not here touch any oval curve; it descends through $M$. below the ellipse $\frac{x^{2}}{a}+\frac{y^{2}}{b}=1$, lying in the upper and lower portions of the hyperboloid, and making round it an infinity of convolutions.

36. We come, then, to the initial direction $M 5$, which is that of the right line; the geodesic here coincides with the right line.

In the cases which follow, the geodesic lies in the upper and lower portions of the hyperboloid, cutting all the oval curves of curvature.

Initial direction $M 6$ : the geodesic does not touch any hyperbolic curve of curvature, but makes round the hyperboloid an infinity of convolutions.

C. VIII. 
Initial direction $M 7$ : the geodesic touches at opposite infinities the mean hyperbola $\frac{x^{2}}{a}+\frac{z^{2}}{c}=1$, it lies wholly in front of the plane $y=0$ of this hyperbola.

Initial direction $M 8$ : the geodesic touches a hyperbolic curve of curvature parameter $q_{8}$ where $q_{8}$ (negative) is between $-b$ and $q_{9}$ the parameter of the hyperbolic curve of curvature through $M$; viz. it cuts all the hyperbolic curves the parameters of which are between $-b$ and $q_{8}$, but does not cut the remaining curves the parameters of which extend from $q_{8}$ to $-a$.

Lastly, initial direction is $M 9$, that of the hyperbolic curve of curvature through $M$; the geodesic touches this curve, cutting all the hyperbolic curves the parameters of which are between $-b$ and $q_{9}$, but not any of those the parameters of which are between $q_{9}$ and $-a$.

37. If in the differential equation of the geodesic line we consider $p, q$ as the elliptic coordinates of a given point $M$ of the curve, the equation for a given value of $\theta$ determines the direction of the curve; or conversely, if the direction be given, the equation determines the value of the parameter $\theta$. Writing

$$
\frac{d p \sqrt{ } p}{\sqrt{(a+p)(b+p)(c+p)}}=P, \quad \frac{d q \sqrt{ } q}{\sqrt{(a+q)(b+q)(c+q)}}=Q
$$

then $P, Q$ are proportional to the rectangular coordinates of a consecutive point $M^{\prime}$, measured from $M$ in the directions of the hyperbolic and oval curves of curvature respectively; and the differential equation of the geodesic lines gives

$$
\frac{P}{\sqrt{p+\theta}} \pm \frac{Q}{\sqrt{q+\theta}}=0
$$

viz. if $\phi$ be the inclination of the geodesic to the hyperbolic curve of curvature, then $Q=P \tan \phi$, or we have $\frac{1}{p+\theta}=\frac{\tan ^{2} \phi}{q+\theta}$, that is, $p \tan ^{2} \phi-q=\theta\left(1-\tan ^{2} \phi\right)$; hence, if for the right line $\phi=\lambda$, then $p \tan ^{2} \lambda-q=0$; and therefore $\theta=\frac{p\left(\tan ^{2} \phi-\tan ^{2} \lambda\right)}{1-\tan ^{2} \phi}$; viz. $\phi=0, \theta=-q,=\infty, \theta=-p$, as it should be. 